Background and Purpose The Pipeline Flex Embolization Device with Shield Technology (PEDS) is an updated version of the Pipeline Flex Embolization Device (PED) which has been modified to include a surface phosphorylcholine biocompatible polymer. It was already reported PED was less effective in recurrent previously stented cerebral aneurysms using Neuroform stent or Enterprise stent than in nonstented aneurysms. However there is no data regarding PEDS for recurrent previously stented cerebral aneurysms using LVIS stent. The aim of this study was to evaluate the efficacy and safety of the PEDS in the treatment of recurrent previously stented aneurysms using LVIS stent.

Materials and Methods We retrospectively analyzed four consecutive patients (mean age, 52.0 years; all female) who underwent PEDS placement for recurrent previously stented cerebral aneurysms using LVIS stent between January 2020 and February 2021. Two aneurysms were located in the internal carotid artery, two aneurysms in the vertebral artery. The mean size of the aneurysms was $18.7 \mathrm{~mm}$ (range, 14-23). Technical success rates, symptomatic stroke within 30 days, occlusion at the latest follow-up angiogram and late adverse events (recurrence and retreatment rates, and strokes or neurologic deaths) were analyzed.

Results The technical success rate was $100 \%$. No symptomatic stroke developed within 30 days. During a mean follow-up period of 5.3 months, complete occlusion was achieved in $3 / 3$ (100\%) and no late adverse events occurred.

Conclusions The PEDS may become treatment option for recurrent previously LVIS stented cerebral aneurysms.

Disclosures K. Takayama: None. T. Wada: None. H. Kishida: None. K. Myouchin: None. K. Kichikawa: None.

\section{E-125 IMPACT OF COVID RELATED RESTRICTIONS ON CLINICAL TRIALS - INSIGHTS FROM THE STERLING REGISTRY}

${ }^{1} \mathrm{R}$ De Leacy ${ }^{*},{ }^{2} \mathrm{~A}$ Puri, ${ }^{3} \mathrm{R}$ Starke, ${ }^{4} \mathrm{~B}$ Jankowitz, ${ }^{5} \mathrm{~A}$ Yoo, ${ }^{6} \mathrm{G}$ Pero, ${ }^{7} \mathrm{C}$ Chivot, ${ }^{8} \mathrm{~T}$ Yao, ${ }^{9} \mathrm{O}$ Zaidat. ${ }^{1}$ Cerebrovascular Center at Mount Sinai, Mount Sinai Health System, New York, NY; ${ }^{2}$ Radiology, Neurological Surgery, and Neurosurgery, UMass Memorial Health Care, Boston, MA; ${ }^{3}$ Neurosurgery, UMiami/Jackson Memorial, Miami, FL; ${ }^{4}$ Neurological Institute, Neurological Surgery, and Stroke Program, Cooper University Health Care, Pittsburgh, PA; ${ }^{5}$ Neurological Surgery, Texas Stroke Institute, Plano, TX; ${ }^{6}$ Neuroradiology, Ospedale Niguarda, Milan, Italy; ${ }^{7}$ Radiology, CHU Amiens Picardie, Amiens, FRANCE; ${ }^{8}$ Neuroscience Institute, Norton Neuroscience Institute, Norton Healthcare, Louisville, $K Y ;{ }^{9}$ Neuroscience Institute, Mercy St. Vincent Hospital, Toledo, $\mathrm{OH}$

\subsection{6/neurintsurg-2021-SNIS.220}

Introduction The STERLING registry is a prospective collection of aneurysms treated with endovascular techniques. The primary intention is to assess the efficacy and safety of Galaxy and MicrusFrame coils (Cerenovus, Irvine, CA). The Covid pandemic has the potential to alter the delivery of medical care or trial enrollment. Changes to regional or hospital specific research infrastructure will likely affect trials for the worse.

Methods A retrospective review of STERLING sites and patients was undertaken to better understand regional variation in adapting to the pandemic and how enrollment was affected.

Results 45 global sites currently take part in the STERLING registry. 28 sites began enrolling pre-Covid. 34\% of U.S. sites were required to halt research, enrollment or elective surgery, whereas only $8 \%$ of EU and none of the Japanese sites were
Abstract E-125 Table 1 Post-COVID differences in actual vs. projected monthly enrollment rates by region

\begin{tabular}{lllll}
\hline Region & $\begin{array}{l}\text { Actual (mean } \pm \\
\text { SD) }\end{array}$ & $\begin{array}{l}\text { Projected(mean } \pm \\
\text { SD) }\end{array}$ & $\begin{array}{l}\text { Mean } \\
\text { Diff. }\end{array}$ & $95 \% \mathrm{Cl}$ \\
\hline US & $5.92 \pm 3.53$ & $9.58 \pm 0.51$ & 3.67 & $1.53 ; 5.80$ \\
EU & $3.83 \pm 2.04$ & $5.83 \pm 1.34$ & 2.00 & $0.54 ; 3.46$ \\
Japan & $1.25 \pm 1.60$ & $2.17 \pm 0.39$ & 0.92 & $-0.07 ;$ \\
& & & & 1.90 \\
Global & $3.67 \pm 1.61$ & $5.86 \pm 0.67$ & 2.19 & $1.15 ; 3.24$ \\
\hline
\end{tabular}

required to significantly change their research protocols respectively. At the 10 (9 US, $1 \mathrm{EU}$ ) sites required to put a hold on research, rates of enrollment dropped from an average of 0.6 subjects/month in the US ( 0.5 in EU) to 0 in the first month and 0.1 subjects/month in the second month of Covid-related restrictions. At the time of this analysis (1/ 2021), the average rate of enrollment across those sites partially recovered to 0.2 subjects/month in the US and 0.2 in EU, although 5 of the 10 sites (all US) had not enrolled any subjects post Covid. Overall, mean actual vs. projected enrollment rates decreased more in the U.S. than in the EU and Japan. The percent of ruptured aneurysms in the per-protocol analysis increased from $23 \%$ to $40 \%$, while the percent of unruptured aneurysms decreased from $76 \%$ to $60 \%$. There were no significant changes to occlusion rates or adverse events pre/ post Covid.

Conclusion The Covid pandemic has the potential to dramatically alter how research is conducted worldwide. U.S. sites faced tougher restrictions than their EU and Japan counterparts. Particularly within the U.S., moratoriums on research and elective surgery may be the driving factor in reducing overall enrollment and increasing the percentage of ruptured aneurysm enrollment. Fortunately, these changes did not appear to negatively affect occlusion rates or adverse events.

Disclosures R. De Leacy: 6; C; Reports other and non-financial support from Penumbra; and non-financial support from Cerenovus and Siemens. A. Puri: 1; C; Has received research grants from Medtronic Neurovascular and Stryker Neurovascular.. 2; C; Serves as a consultant for Medtronic Neurovascular Stryker Neurovascular. R. Starke: 2; C; Serves as a consultant for for Medtronic Neurovascular, Penumbra, Cerenovus, and Abbott. B. Jankowitz: 2; C; Serves as a consultant for Medtronic and Stryker. A. Yoo: 1; C; Receives research grants from Medtronic, Cerenovus, Penumbra, Stryker, and Genentech. 2; C; Serves as a consultant for Cerenovus, Penumbra, and Zoll.. 4; C; Has equity interest in Insera Therapeutics. G. Pero: None. C. Chivot: None. T. Yao: 2; C; Serves as a consultant/proctor for Medtronic. O. Zaidat: 2; C; Serves as a consultant for Neuravi, Stryker, Penumbra, and Medtronic.

\section{E-126 COMPARISON OF STENT RETRIEVER THROMBECTOMY USING 3D PATIENT SPECIFIC MODELS OF INTRACRANIAL CIRCULATION WITH ACTUAL MIDDLE CEREBRAL ARTERY OCCLUSION THROMBECTOMY CASES}

${ }^{1} \mathrm{M}$ Mokin*, ${ }^{1}$ E Pressman, ${ }^{2} \mathrm{~K}$ Sommer, ${ }^{2} \mathrm{C}$ Ionita. ${ }^{1}$ University of South Florida, Tampa, FL; ${ }^{2}$ University at Buffalo, Buffalo, NY

10.1136/neurintsurg-2021-SNIS.221 